\title{
Mismatch Compensation Technique for Inverter-Based CMOS Circuits
}

DOI:

10.1109/ISCAS.2018.8351057

\section{Document Version}

Accepted author manuscript

Link to publication record in Manchester Research Explorer

\section{Citation for published version (APA):}

Mroszczyk, P., \& Pavlidis, V. (2018). Mismatch Compensation Technique for Inverter-Based CMOS Circuits. In IEEE International Symposium on Circuits and Systems https://doi.org/10.1109/ISCAS.2018.8351057

\section{Published in:}

IEEE International Symposium on Circuits and Systems

\section{Citing this paper}

Please note that where the full-text provided on Manchester Research Explorer is the Author Accepted Manuscript or Proof version this may differ from the final Published version. If citing, it is advised that you check and use the publisher's definitive version.

\section{General rights}

Copyright and moral rights for the publications made accessible in the Research Explorer are retained by the authors and/or other copyright owners and it is a condition of accessing publications that users recognise and abide by the legal requirements associated with these rights.

\section{Takedown policy}

If you believe that this document breaches copyright please refer to the University of Manchester's Takedown Procedures [http://man.ac.uk/04Y6Bo] or contact uml.scholarlycommunications@manchester.ac.uk providing relevant details, so we can investigate your claim.

\section{OPEN ACCESS}




\section{Mismatch Compensation Technique for Inverter-Based CMOS Circuits}

\author{
Przemyslaw Mroszczyk \\ Advanced Processor Technologies Group \\ School of Computer Science, The University of Manchester, \\ Manchester, UK
}

\begin{abstract}
Inverter-based CMOS circuits are often considered in the front-end modules for optical and wireline communication, A-D conversion, and analogue computation. Due to parameter variability (mismatch), the performance of such circuits is usually degraded. This paper presents a mismatch compensation technique employing a set of redundant switches to trim the switching threshold of inverter-based CMOS circuits. Over 10× better parameter matching is observed at no additional energy cost or significant gate area increase, compared to "traditional" geometry scaling. The efficiency of the mismatch compensation is investigated across a broad design space considering the number and size of the switches, and the size of the inverter, using models from a $65 \mathrm{~nm}$ CMOS technology. The case study of a comparator circuit is further investigated in terms of the reliability, energy, and area, and compared against the geometry scaling approach.
\end{abstract}

Keywords—parameter variability; mismatch; trimming; CMOS inverter; comparator

\section{INTRODUCTION}

A CMOS inverter is perhaps the simplest structure commonly used in integrated circuit design. In addition to digital systems, inverters are often present at the heart of many functional blocks, such as high speed amplifiers, comparators, delay lines, and are also used in analogue computation and neuromorphic circuits [1]-[5]. Due to the very simple topology of this two transistor push-pull amplifier, the area and energy requirements of an inverter are usually minimal with respect to the high gain bandwidth and speed of an inverter-based circuit. Therefore, a CMOS inverter is frequently considered the most efficient circuit in a given technology [6].

An inherent shortcoming of a CMOS process is a certain level of random variability of the circuit parameters known as manufacturing mismatch. As a result, the key parameters of an inverter such as gain, propagation delay, and switching threshold cannot be precisely determined at the design stage. This uncertainty often favors "tried-and-tested" solutions using less efficient and more complex circuits than an inverter, significantly trading area, energy efficiency, and speed for reliability [7].

The method most commonly used to mitigate mismatch is geometry scaling. This approach is based on the fact that the variability of the physical and electrical parameters in CMOS technologies reduces as the device area increases [8]. However, parameter matching improves only with the square root of the

This project has received funding from the European Union's Horizon 2020 research and innovation programme under grant agreement No 671578 .

\author{
Vasilis F. Pavlidis \\ Advanced Processor Technologies Group \\ School of Computer Science, The University of Manchester, \\ Manchester, UK
}

device area, and the required size (and gate capacitance) of an inverter, resilient to process variability, is prohibitively large. Therefore, applications of these circuits are limited to highspeed front-end amplifiers in optical and radio frequency (RF) transceivers, where large devices are typically used to meet gain bandwidth and high driving strength requirements [1]

One of the most area efficient approaches aiming at random offset voltage cancellation, resulting from parameter mismatch, employs auto-zero compensation. However, this technique is limited to circuits allowing discrete time operation [3], [4]. The majority of applications, where the inherent area and power efficiency of an inverter is of a great advantage, usually require continuous time circuits.

Another technique used to improve parameter matching, applicable both to the continuous and discreet time circuits, employs trimming. In this approach, a set of redundant devices (e.g. transistors or resistors) is selectively connected or disconnected from the circuit, "trimming" its operation to counteract mismatch. Despite the proven efficiency of this technique in mismatch mitigation, circuit benchmarking and search for the optimum solution across a large space of possible circuit configurations is required. Due to the high complexity, the trimming processes are usually conducted externally increasing the implementation cost and limiting the flexibility of the designed system. Therefore, trimming is usually considered in applications where energy efficiency and precision are paramount [9], [10].

Recognizing the advantages of the trimming technique, this paper presents the application of trimming for mismatch compensation in inverter-based circuits. Rather than enlarging transistors to reduce the variability of the gain, propagation delay, and switching threshold, a set of redundant, small size MOS switches is used to "balance" the strengths of the pull-up and pull-down networks, thereby counteracting the effects of the mismatch. Addressing the aforementioned challenges, the proposed trimming technique autonomously finds the best circuit configuration while requiring only a shift register. The approach offers superior mismatch compensation compared to "traditional" geometry scaling. The paper explores the effect of the number and size of the additional drivers on mismatch compensation in the inverters with different transistor widths.

The paper consists of five sections. The proposed trimming technique is presented in Section II. The related simulation results are discussed in Section III. The example comparator 
circuit is described in Section IV. Conclusions are provided in Section V.

\section{INVERTER TRIMMING}

This section presents the proposed trimming technique, the circuit implementation, and a dedicated test circuit used in a mixed-signal simulation environment.

\section{A. Technique}

The schematic diagram of the inverter with the circuit allowing switching threshold adjustment is illustrated in Fig. 1. The switching threshold $\mathrm{V}_{\mathrm{ST}}$ is defined here as the crossover point on the DC transfer characteristic of an inverter where the input and output voltages are equal, also known as the point of the highest inverter sensitivity. The circuit consists of an inverter comprising transistors $\mathrm{M}_{\mathrm{N}}$ and $\mathrm{M}_{\mathrm{P}}$ and a set of additional drivers $\left(\mathrm{M}_{\mathrm{N} 1}-\mathrm{M}_{\mathrm{N} 8}\right.$ and $\left.\mathrm{M}_{\mathrm{P} 1}-\mathrm{M}_{\mathrm{P} 8}\right)$ with individual switches $\left(\mathrm{M}_{\mathrm{SN} 1}-\mathrm{M}_{\mathrm{SN} 8}\right.$ and $\left.\mathrm{M}_{\mathrm{SP} 1}-\mathrm{M}_{\mathrm{SP} 8}\right)$. These additional transistors are selectively used to "balance" the driving strength of the $M_{N}$ and $M_{P}$ networks for a given reference voltage $V_{R E F}$. The trimming procedure aims at finding a configuration of switches ensuring that the point of the highest sensitivity occurs for a given input bias $\mathrm{V}_{\mathrm{REF}}$, such that $\mathrm{V}_{\mathrm{ST}}=\mathrm{V}_{\mathrm{REF}}$. The additional drivers are set to the minimum size while their strength is controlled through geometry of the switches. This choice enables keeping the input capacitance of the inverter to the minimum.

The transfer characteristic of the circuit is shown in Fig. 2. Note that the input voltage is constant $\left(\mathrm{V}_{\mathrm{REF}}\right)$ while the output voltage changes as a result of altering the logic states of the signals controlling the switches. For all the signals set to logic " 1 " (defined as CODE =0), the n-MOS switches are in the ON state, the p-MOS switches are in the OFF state. With this configuration, the driving strength of the $\mathrm{M}_{\mathrm{N}}$ network is the highest, the driving strength of the $M_{P}$ network is the lowest, and the output voltage reaches the minimum value $\mathrm{V}_{\text {LOW. }}$. By turning signals $\mathrm{S}_{\mathrm{N} 1}-\mathrm{S}_{\mathrm{N} 8}$ successively to logic "0", the corresponding n-MOS drivers switch off, gradually decreasing the strength of the $\mathrm{M}_{\mathrm{N}}$ network increasing the switching threshold $\mathrm{V}_{\mathrm{ST}}$ of the inverter. The encoding scheme showing the relation between the codes and the configurations of the switches $\left[\mathrm{S}_{\mathrm{N} 1} \ldots \mathrm{S}_{\mathrm{N} 8}, \mathrm{~S}_{\mathrm{P} 1}, \ldots \mathrm{S}_{\mathrm{P} 8}\right]$, is listed in Table I. Note that this encoding scheme ensures a monotonic shift of the $\mathrm{V}_{\mathrm{ST}}$, despite the parameter mismatch, while the consecutive codes are generated and stored in a shift register, which significantly simplifies the circuit realization.

\section{B. Circuit implementation}

The schematic diagram of the trimming circuit is shown in Fig. 3, and the waveforms are depicted in Fig. 4. The circuit consists of an amplifier with 4 cascaded inverters and a 16-bit shift register used for code generation. Prior to trimming, all the bits are set to " 1 ", equivalent to the first configuration, i.e. $\mathrm{CODE}=0$. The consecutive codes are generated on each rising edge of the CLK signal and the output voltage (see Fig. 2) of the first inverter in the amplifier increases. Once this voltage crosses the switching threshold of the second inverter, the output states of the remaining inverters change accordingly. As

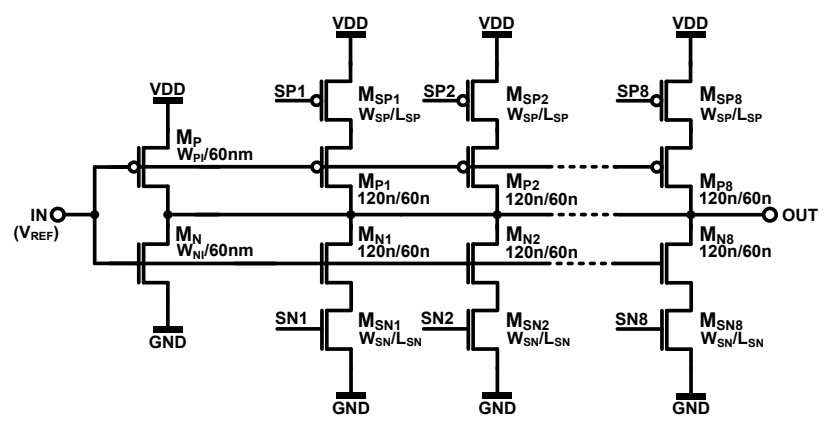

Fig. 1. The schematic diagram of an inverter with 8 trimming stages.

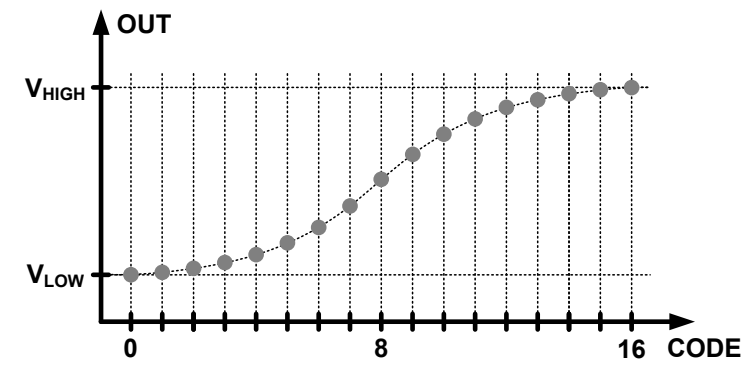

Fig. 2. The output voltage of the inverter in response to the monotonic sweep of the trimming codes.

TABLE I. ENCODING SCHEME OF THE CONFIGURATION SWITCHES

\begin{tabular}{c|c}
\hline \hline CODE & Vector $\left[\mathbf{S}_{\mathbf{N} 1} \ldots \mathbf{S}_{\mathbf{N} 8}, \mathbf{S}_{\mathbf{P 1}}, \ldots \mathbf{S}_{\mathbf{P 8}}\right]$ \\
\hline 0 & {$[11111111111111]$} \\
\hline 1 & {$[01111111111111]$} \\
\hline 2 & {$[001111111111111]$} \\
\hline 8 & {$[000000001111111]$} \\
\hline 15 & {$[0000000000000001]$} \\
\hline 16 & {$[0000000000000000]$} \\
\hline \hline
\end{tabular}

a result, the output of the amplifier will transition from the high to the low logic state, disabling the clock input of the D-type flip flops and, hence, locking the current code. If $\mathrm{V}_{\mathrm{REF}}$ is beyond the trimming range, the circuit locks on the first or on the last code always minimizing the difference between $V_{\text {REF }}$ and the actual value of $\mathrm{V}_{\mathrm{ST}}$. Note that the trimming is applied only to the first inverter stage. This feature has practically negligible impact on the trimming efficiency since the

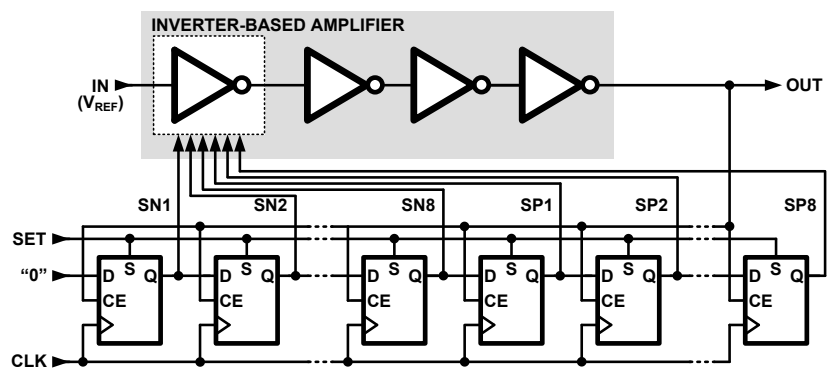

Fig. 3. Schematic diagram of the trimming circuit with amplifier.

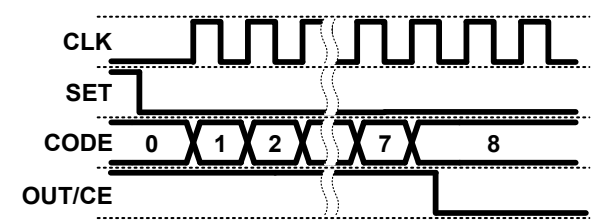

Fig. 4. Signal waveforms of the trimming process. 
amplitude of the signal quickly increases far beyond the variability of the switching threshold of the inverters in the chain.

\section{Test circuit}

The circuit used in the simulations is depicted in Fig. 5. The inverter-based amplifier is implemented in a $65 \mathrm{~nm}$ CMOS technology with layout-estimated parasitics. The configuration of the switches is determined by the digital controller based on the state of the amplifier output (MODE 0). Once the trimming is complete, the analogue multiplexer and 16-bit rail-to-rail DAC are used in the evaluation of the switching threshold $\mathrm{V}_{\mathrm{ST}}$ employing the successive approximation method (MODE 1). Note that the multiplexer and DAC are used for the quick $V_{S T}$ estimation and are part of the testbench, thus Verilog-AMS models are preferred to shorten the simulation time. In MODE 2 , the amplifier is used for performance tests (see Section IV).

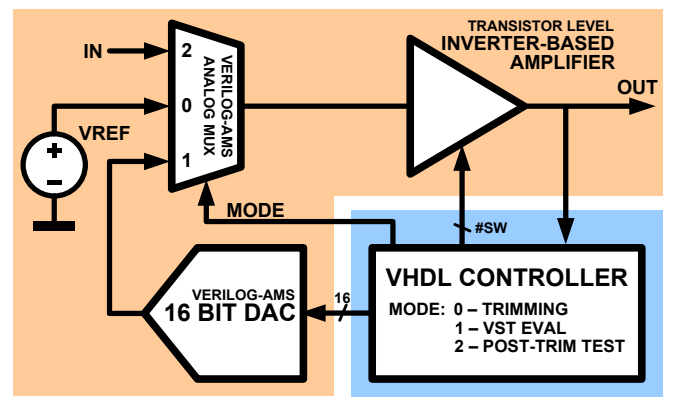

Fig. 5. The block diagram of the test circuit.

\section{SIMULATION RESULTS}

This section presents the Monte Carlo simulation results of the $\mathrm{V}_{\mathrm{ST}}$ variability of the inverter-based amplifier designed for $\mathrm{V}_{\mathrm{ST}}=\mathrm{V}_{\mathrm{DD}} / 2=600 \mathrm{mV}$. The reference values of the circuit parameters are listed in Table II. The trimming sequence and the $\mathrm{V}_{\mathrm{ST}}$ are simulated assuming parameter mismatch at nominal operating conditions ( $\mathrm{TT}$ process corner, $\mathrm{V}_{\mathrm{DD}}=1.2 \mathrm{~V}, \mathrm{~T}=$ $25^{\circ} \mathrm{C}$ ). Note that, the systematic shift of the $V_{S T}$ in other process, voltage, and temperature (PVT) corners can be compensated for by the circuit as long as a sufficiently large trimming range is ensured.

The trimming efficiency in terms of the size of the input stage transistors $\mathrm{M}_{\mathrm{N}}$ and $\mathrm{M}_{\mathrm{P}}$ for different number of trimming stages is shown in Fig. 6. The trimming efficiency is defined as the ratio $\sigma_{\mathrm{VST}-\mathrm{G}} / \sigma_{\mathrm{VST}-\mathrm{T}}$. The $\sigma_{\mathrm{VST}-\mathrm{T}}$ is the standard deviation of the $\mathrm{V}_{\mathrm{ST}}$ of the amplifier with trimming (Fig. 3). The $\sigma_{\mathrm{VST}-\mathrm{G}}$ is the standard deviation of the $\mathrm{V}_{\mathrm{ST}}$ of the same circuit without trimming stages but with the geometry (width) of the $\mathrm{M}_{\mathrm{P}}\left(\mathrm{M}_{\mathrm{N}}\right)$ enlarged accordingly to ensure the same total gate area of the input stage as in the circuit with trimming. Note that the maximum trimming efficiency occurs only for a specific size of the input stage transistors. When $\mathrm{W}_{\mathrm{P}}\left(\mathrm{W}_{\mathrm{N}}\right)$ is small, the effect of the additional drivers on $\mathrm{M}_{\mathrm{P}}\left(\mathrm{M}_{\mathrm{N}}\right)$ is higher, the intervals between the neighboring codes expand (the trimming granularity increases), and the trimming precision degrades increasing $\sigma_{\text {VST-T }}$ which reduces the ratio $\sigma_{\text {VST-G }} / \sigma_{\text {VST-T }}$ (see the left hand side slopes of the traces in Fig. 6). When $W_{P}\left(W_{N}\right)$ is larger, the impact of the additional drivers on $M_{P}\left(M_{N}\right)$
TABLE II. REFERENCE VALUES OF THE CIRCUIT PARAMETERS

\begin{tabular}{c|c}
\hline \hline Parameter & Value \\
\hline $\mathrm{W}_{\mathrm{P}} / \mathrm{W}_{\mathrm{N}}$ & $3 \mu \mathrm{m} / 1 \mu \mathrm{m}$ \\
\hline $\mathrm{W}_{\mathrm{SP}} / \mathrm{L}_{\mathrm{SP}}$ & $120 \mathrm{~nm} / 200 \mathrm{~nm}$ \\
\hline $\mathrm{W}_{\mathrm{SN}} / \mathrm{L}_{\mathrm{SN}}$ & $120 \mathrm{~nm} / 2 \mu \mathrm{m}$ \\
\hline $\mathrm{V}_{\mathrm{REF}} / \mathrm{V}_{\mathrm{DD}}$ & $600 \mathrm{mV} / 1.2 \mathrm{~V}$ \\
\hline \hline
\end{tabular}

becomes less significant and $\sigma_{\mathrm{VST}-\mathrm{T}}$ converges to $\sigma_{\mathrm{VST}-\mathrm{G}}$ (see the right hand side slopes of the traces in Fig. 6).

The simulation results of a circuit with a fixed number of 8 trimming stages and different driving strengths are presented in Fig. 7. Note that the efficiency trace "shrinks" or "expands" along the $\mathrm{W}_{\mathrm{P}}\left(\mathrm{W}_{\mathrm{N}}\right)$ axis as the strength of the trimming stages changes. Thus, the maximum efficiency occurs for a specific ratio of the $\mathrm{W}_{\mathrm{P}}\left(\mathrm{W}_{\mathrm{N}}\right)$ strength and the strength of the trimming stages and, hence, can be decided by adjusting this ratio at the design stage, e.g. by appropriate sizing of the switches.

The absolute values of the standard deviation $\left(\sigma_{\mathrm{VST}-\mathrm{T}}\right)$ and the mean value $\left(\mu_{\mathrm{VST}-\mathrm{T}}\right)$ in terms of $\mathrm{V}_{\mathrm{REF}}$ in the range $580 \mathrm{mV}$ $620 \mathrm{mV}$ are shown in Fig. 8 and 9 respectively. Note that increasing the number of trimming stages the trimming range also extends. While an amplifier with 6 trimming stages allows

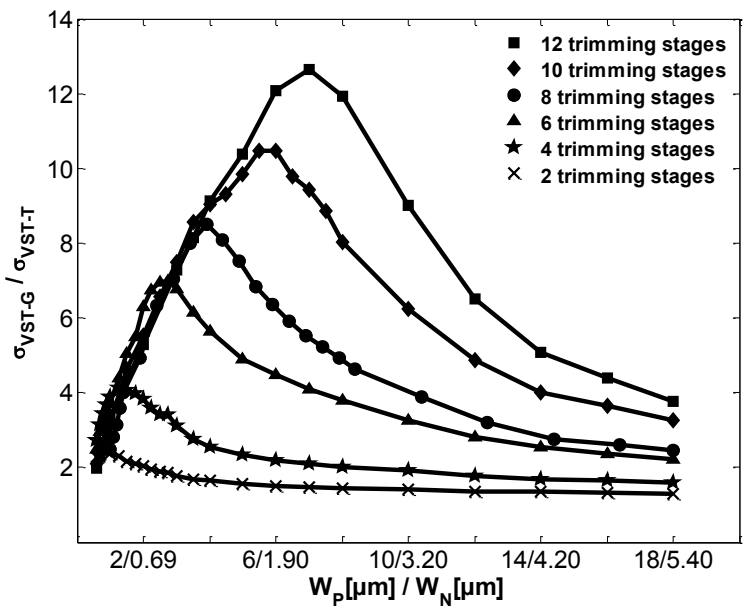

Fig. 6. Trimming efficiency in terms of the size of the input stage transistors $\mathrm{M}_{\mathrm{P}}$ and $\mathrm{M}_{\mathrm{N}}$ for different number of trimming stages.

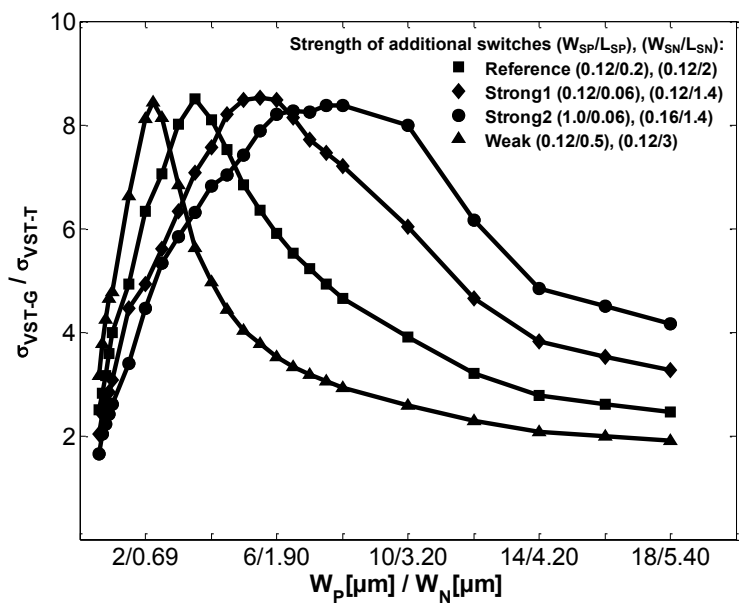

Fig. 7. Trimming efficiency in terms of the size of the input stage transistors $\mathrm{M}_{\mathrm{P}}$ and $\mathrm{M}_{\mathrm{N}}$ for different sizes of the switches in the input stage amplifier. 
$\sigma_{\mathrm{VST}-\mathrm{T}} \approx 750 \mu \mathrm{V}$ only for $\mathrm{V}_{\mathrm{REF}}=600 \mathrm{mV}$, the same amplifier with 12 stages can be trimmed in the range of $\mathrm{V}_{\mathrm{REF}}+/-10 \mathrm{mV}$.

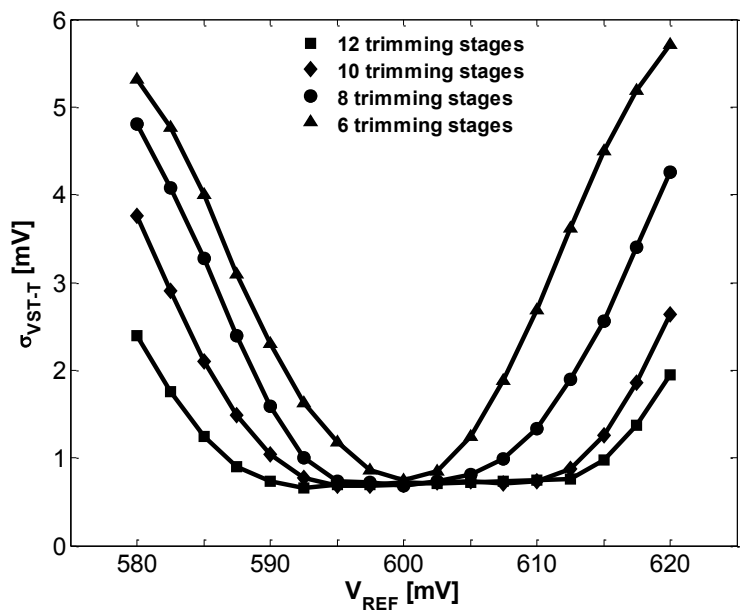

Fig. 8. Standard deviation of the $\mathrm{V}_{\mathrm{ST}}$ after trimming in terms of the reference $\mathrm{V}_{\mathrm{REF}}$ for different number of switches of the input stage amplifier.

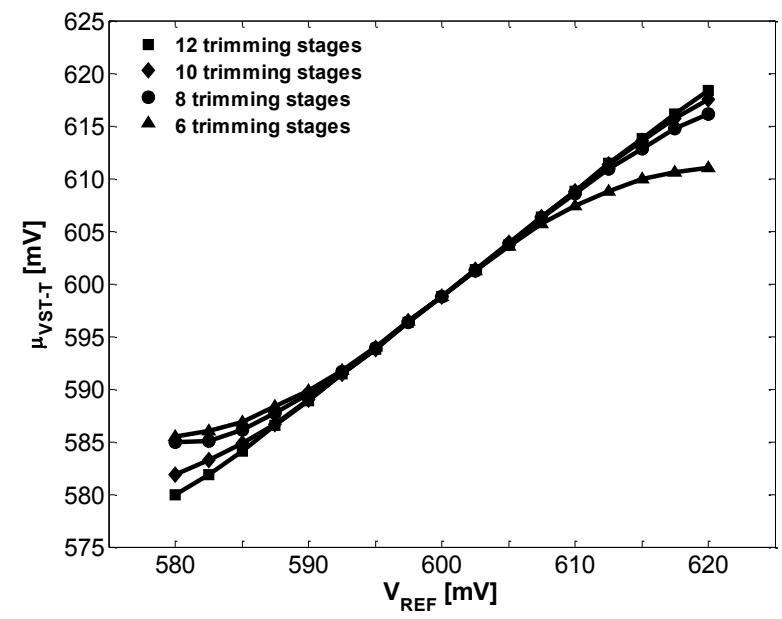

Fig. 9. Mean value of the $\mathrm{V}_{\mathrm{ST}}$ after trimming in terms of the reference $\mathrm{V}_{\mathrm{REF}}$ for different number of switches of the input stage amplifier.

\section{INVERTER-BASED COMPARATOR}

This section presents an application of the inverter-based amplifier (Fig. 3) as a high speed comparator. The test circuit used in the simulations is shown in Fig. 10. It consists of the inverter-based amplifier with 8 trimming stages with reference circuit parameters from Table II, the pseudo-random bit stream generator (PRBS), and the bit error rate test module (BER). The PRBS generates a bit sequence at $1 \mathrm{~Gb} / \mathrm{s}$ speed around the reference $\mathrm{V}_{\mathrm{REF}}+/-\Delta \mathrm{V}_{\text {IN }}$. The BER test module compares the input and output signals of the comparator on bit-to-bit bases and counts the number of encountered errors. The output of the comparator is recognized as logic " 0 " or " 1 " when the sampled output voltage ( $100 \mathrm{ps}$ prior to the next bit) is below $300 \mathrm{mV}$ or over $900 \mathrm{mV}$, respectively, otherwise an error is recorded. The bit error rate for a given input swing $\Delta \mathrm{V}_{\mathrm{IN}}$ is estimated based on 100 Monte Carlo simulation runs including trimming and test (MODE 2) with a random sequence of 1000 bits. The BER in terms of $\Delta \mathrm{V}_{\mathrm{IN}}$ is shown in Fig. 11. For comparison, BER of three comparators with no trimming and increased size of the

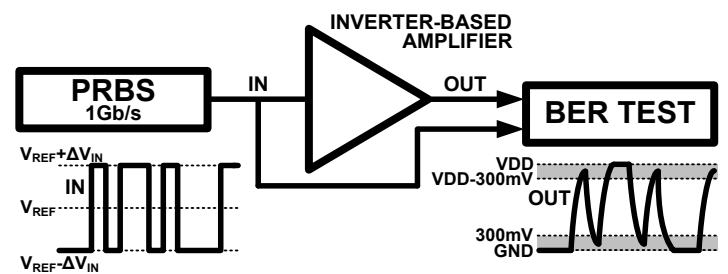

Fig. 10. Test circuit used in the simulation of the inverter-based comparator.

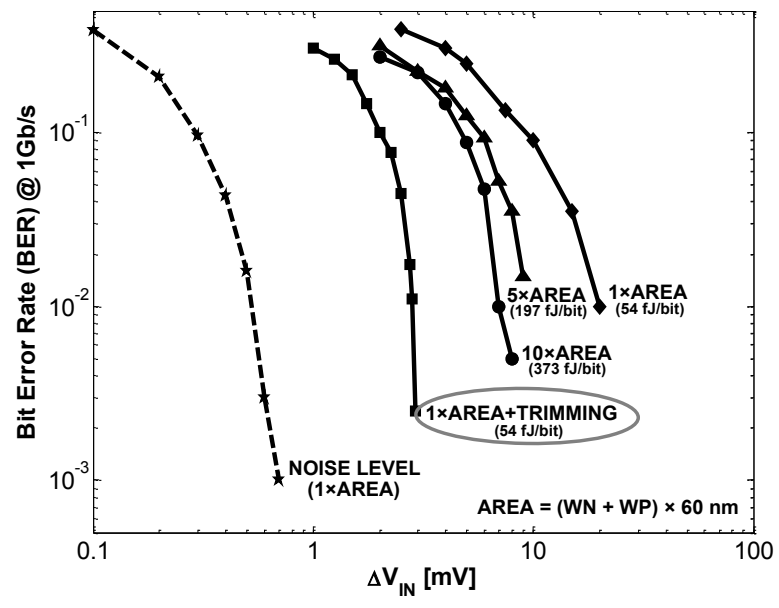

Fig. 11. Bit error rate vs. $\Delta \mathrm{V}_{\mathrm{IN}}$ voltage of the inverter-based comparator illustrating performance under mismatch, noise and geometry scaling.

input stage transistors by $1 \times, 5 \times$, and $10 \times$ the reference area from Table II are provided. Noise induced BER is evaluated in transient noise simulations of the same test circuit for $20 \mathrm{GHz}$ bandwidth assuming no variability.

The proposed trimming technique achieves about $10 \times$ lower BER than the same circuit without trimming, at no additional energy cost. Moreover, the performance of the proposed circuit cannot be achieved through geometry scaling without a significant increase in the area and power of the comparator. Note that $10 \times$ the area increase in the scaling approach entails $7 \times$ higher power consumption while reducing BER only by factor of 3 . Further BER reduction is possible by using more trimming stages, however, limited by noise when $\Delta \mathrm{V}_{\mathrm{IN}}<1 \mathrm{mV}$. The energy efficiency of the circuit can be improved by reducing the size of the input stage transistors and by implementing other low power techniques (e.g. supply voltage reduction) [3]. In addition, inverter-based comparators exhibit very small kickback [11] due to a low voltage gain of the first stage. Also, the information about the switching threshold is stored in the shift register and the reference can be powered off after the trimming, which is beneficial to some applications with low power requirements such as Flash ADCs.

\section{CONCLUSIONS}

In this paper, a novel approach to mitigate mismatch in inverter-based CMOS circuits is presented. The proposed technique achieves significantly better parameter matching at no additional energy cost. Due to the small gate area, small input capacitance, and high energy efficiency, the inverterbased circuits with trimming are suitable for a variety of applications such as dc-coupled sensor amplifiers, front-end receivers, and low power ADCs. 


\section{REFERENCES}

[1] K. Park and W. S. Oh, "A 40-Gb/s 310-fJ/b Inverter-Based CMOS Optical Receiver Front-End," IEEE Photonics Technology Letters, Vol. 27, No. 18, pp. 1931 - 1933, June 2015.

[2] K. Eguchi et al., "A Median Filtering Circuit Using Clocked CMOS Neuron Inverters for Implantable Electronic Medical Devices,' International Journal of Innovative Computing, Information and Control, Vol. 13, No. 4, pp. 1135 - 1147, August 2017.

[3] H. Lee, "A Low-Voltage Low-Power Comparator with CurrentControlled Dynamically-Biased Preamplifiers for DCM Buck Regulators," Proceedings of the International Conference on Electronics, Circuits, and Systems, pp. 371 - 374, December 2009.

[4] T. Sugiki et al., "A $60 \mathrm{~mW} 10$ b CMOS Image Sensor with Column-toColumn FPN Reduction," Proceedings of the IEEE International SolidState Circuits Conference, February 2000.

[5] P. Mroszczyk and P. Dudek, "Tunable CMOS Delay Gate With Improved Matching Properties," IEEE Transactions on Circuits and Systems I, Vol. 61, No. 9, pp. 2586 - 2595, September 2014.

[6] C. Svensson, "Optimum Voltage Swing on On-Chip and Off-Chip Interconnect," IEEE Journal of Solid State Circuits, Vol. 36, No. 7, pp. 1108-1112, July 2001.

[7] P. Kinget, "Device Mismatch and Tradeoffs in the Design of Analog Circuits," IEEE Journal of Solid-State Circuits, Vol. 40, No. 6, pp. 1212 - 1224, June 2005.

[8] M. J. M. Pelgrom, A. C. J. Duinmaijer, and A. P. G. Welbers, "Matching Properties of MOS Transistors", IEEE Journal of Solid-State Circuits, Vol. 24, No. 5, pp. 1433 - 1439, October 1989.

[9] X. Yang and J. Liu, "A 10 GS/s 6 b Time-Interleaved Partially Active Flash ADC," IEEE Transactions on Circuits and Systems I, Vol. 61, No. 8 , pp. 2272 - 2280, August 2014.

[10] E. H. M. Heijne et al., "LHC1: A Semiconductor Pixel Detector Readout Chip with Internal, Tunable Delay Providing a Binary Pattern of Selected Events," Elsevier Nuclear Instruments and Methods in Physics Research, Section A: Accelerators, Spectrometers, Detectors and Associated Equipment, Vol. 383, No. 1, pp. 55 - 63, December 1996.

[11] P. M. Figueiredo, J C. Vital, "Kickback Noise Reduction Techniques for CMOS Latched Comparators," IEEE Transactions on Circuits and Systems II, Vol. 53, No. 7, pp. 541 - 545, July 2006. 